\title{
Simvastatin improves intracerebral hemorrhage through NF-kB-mediated apoptosis via the MyD88/TRIF signaling pathway
}

\author{
CHENGYAO GU $^{1}$, YUNQIN WU ${ }^{1}$, ZHENYI FAN ${ }^{1}$ and WEIWEI HAN ${ }^{2}$ \\ Departments of ${ }^{1}$ Neurology and ${ }^{2}$ Rehabilitation, Ningbo No. 2 Hospital, Ningbo, Zhejiang 315010, P.R. China
}

Received June 10, 2017; Accepted August 4, 2017

DOI: $10.3892 /$ etm.2017.5349

\begin{abstract}
The aim was to investigate the neuroprotective effects and potential mechanism mediated by simvastatin in a mouse model of intracerebral hemorrhage. CD-1 mice were subjected to infusion of collagenase type IV into the left striatum in order to induce intracerebral hemorrhage. Western blot analysis, the TUNEL assay and the modified neurological severity score were used in the present study to analyze the efficacy of simvastatin for intracerebral hemorrhage. The results demonstrated that simvastatin treatment improved the cerebral water content and blood-brain barrier disruption in the intracerebral hemorrhage animals. Intracerebral hemorrhage-induced neuronal cell death was downregulated by simvastatin treatment compared with the vehicle-treated model group. In addition, the expression levels of aquaporin-4, matrix metallopeptidase 9 and caspase-3 were downregulated and B-cell lymphoma-2 was upregulated by simvastatin treatment compared with the vehicle-treated model. Simvastatin treatment also significantly reduced the Evans blue leakage into the injured hemispheres and improved motor function. Mechanism analysis further indicated that simvastatin treatment downregulated nuclear factor $(\mathrm{NF})-\mathrm{\kappa B}$ expression, and upregulated the myeloid differentiation primary response 88 (MyD88) and TIR domain-containing adaptor protein inducing interferon- $\beta$ (TRIF) expression levels in neuronal cells in experimental mice. Furthermore, the results revealed that NF- $\mathrm{KB}$ overexpression abolished the simvastatin-downregulated MyD88 and TRIF expression levels, as well as the apoptosis of neuronal cells. In conclusion, these results indicated that simvastatin was able to attenuate brain edema and reduce cellular apoptosis by suppressing the NF- $\kappa \mathrm{B}$-mediated MyD88/TRIF signaling pathway subsequent to the induction of intracerebral hemorrhage in mice.
\end{abstract}

Correspondence to: Dr Weiwei Han, Department of Rehabilitation, Ningbo No. 2 Hospital, 41 Xi'bei Street, Ningbo, Zhejiang 315010, P.R. China

E-mail: xiaohan0776@sina.com

Key words: intracerebral hemorrhage, simvastatin, apoptosis, nuclear factor- $\kappa \mathrm{B}$, myeloid differentiation primary response 88 , TIR domain-containing adaptor protein inducing interferon- $\beta$

\section{Introduction}

Intracerebral hemorrhage is the most common human cerebrovascular disease and the subtype of hemorrhagic stroke that is most difficult to treat (1). Patients with acute intracerebral hemorrhage were frequently monitored in the intensive care unit $(2,3)$. Spontaneously sudden intracerebral hemorrhage is associated with higher rates of mortality and morbidity in comparison with other intracephalic diseases (4). Cerebral hemorrhage is frequently induced by exertion and emotion, and the majority of patients demonstrate sudden onset during activity (5). In addition, cerebral hemorrhage usually causes severe dysfunction of the cerebral nervous system and loss of working and self-care abilities, consequently further increasing the burden of the patient's family $(6,7)$. Previous studies have observed that the neuroprotective functions contributed to the downregulation of inflammatory (CD68-positive) cells in a mouse model of intracerebral hemorrhage $(2,8)$.

Simvastatin is a statin drug used to manage the blood cholesterol levels and prevent cardiovascular disease due to its inhibitory effect on 3-hydroxy-3-methylglutaryl coenzyme A reductase $(9,10)$. To date, simvastatin has been reported to increase the survival of septic or infectious patients by improvement of the sepsis-induced mortality and acute kidney injury via its renal vascular effects (11). Karki et al (12) have demonstrated that simvastatin and atorvastatin significantly improved neurological recovery, decreased tissue loss and increased neurogenesis when administered for 1 week following intracerebral hemorrhage. In addition, Lapchak and Han (13) have suggested that the 3-hydroxy-3-methylglutaryl coenzyme A reductase inhibitor simvastatin was able to reduce the thrombolytic-induced intracerebral hemorrhage in embolized rabbits. Furthermore, another study indicated that vascular recovery was promoted by simvastatin following experimental intracerebral hemorrhage, as determined by magnetic resonance imaging and histological examinations (14). Therefore, simvastatin may be an efficient agent for intracerebral hemorrhage therapy.

In the present study, the anti-apoptotic properties of simvastatin in the progression of intracerebral hemorrhage were investigated in a mouse model. It was hypothesized that simvastatin may be able to protect neurons by regulating neuronal apoptosis. The study reports that simvastatin led to the attenuation of brain edema and reduced cellular 
apoptosis by suppressing the nuclear factor (NF)- $\mathrm{B}$-mediated myeloid differentiation primary response 88 (MyD88)/TIR domain-containing adaptor protein inducing interferon- $\beta$ (TRIF) signaling pathway subsequent to the induction of intracerebral hemorrhage in mice.

\section{Materials and methods}

Ethical statement. All animal protocols were performed in accordance with the recommendations of the Guide for the Care and Use of Laboratory Animals of the National Institutes of Health (Bethesda, MD, USA). Experiments were approved by the Committee on the Ethics of Animal Experiments Defense Research of Ningbo No. 2 Hospital (Ningbo, China).

Intracerebral hemorrhage animal model. Intracerebral hemorrhage was established using the stereotaxic intrastriatal injection of collagenase type IV as described previously with certain modifications (15). Briefly, a total of 60 CD-1 mice (6-8 weeks old; 300-350 g body weight) were purchased from the Institute of Biophysics at the Chinese Academy of Sciences (Beijing, China), and housed in a temperature-controlled facility at $23 \pm 1^{\circ} \mathrm{C}$ with relative humidity of $50 \pm 5 \%$ and a $12 \mathrm{~h} \mathrm{light/dark} \mathrm{cycle.} \mathrm{The} \mathrm{mice}$ were anesthetized with $10 \%$ chloral hydrate $(0.3 \mathrm{ml} / 100 \mathrm{~g}$, intraperitoneally; Sigma-Aldrich; Merck KGaA, Darmstadt, Germany). The rectal temperature was maintained at $37^{\circ} \mathrm{C}$ throughout the surgical procedure using a heating lamp. Collagenase type IV (0.5 IU; Sigma-Aldrich; Merck KGaA) in $2 \mu \mathrm{l}$ saline was injected into the left striatum to induce intracerebral hemorrhage over a period of $5 \mathrm{~min}$. Mice were divided into two groups (the model + vehicle group and the model + simvastatin group; $n=30$ in each group) and intracerebral hemorrhage animals received intragastric administration of the vehicle (vehicle-treated model) or simvastatin (50 $\mu \mathrm{g}$ dissolved in $500 \mu \mathrm{l}$ saline) once a day immediately following intracerebral hemorrhage.

Western blot analysis. Following treatment with simvastatin for 10 days, the experimental animals were sacrificed and neuronal cells were isolated as previously described (16). Cells were collected and lysed in a radioimmunoprecipitation assay buffer (Thermo Fisher Scientific, Inc., Waltham, MA, USA) followed homogenization at $4{ }^{\circ} \mathrm{C}$ for $10 \mathrm{~min}$. Protein concentration was measured using a BCA kit (cat no. 23225; Thermo Fisher Scientific, Inc.). A total of $20 \mu \mathrm{g}$ protein extract underwent $12.5 \%$ SDS-PAGE and was then transferred to a nitrocellulose membrane. The membrane was incubated in blocking buffer $(5 \%$ milk) for $30 \mathrm{~min}$ at $37^{\circ} \mathrm{C}$ prior to incubation with primary antibodies at $4^{\circ} \mathrm{C}$ overnight. The primary rabbit anti-mouse antibodies used in the immunoblotting assays were as follows: NF- $\mathrm{B}(1: 1,200$; ab32360), aquaporin-4 (AQP4; 1:1,200; ab9512), matrix metallopeptidase 9 (MMP-9; 1:1,000; ab54230), caspase-3 (1:1,200; ab2171), B-cell lymphoma-2 (Bcl-2; 1:1,000; ab692), MyD88 (1:1,200; ab2068), TRIF (1:1,200; ab13810) and $\beta$-actin (1:500; ab8226; all from Abcam, Cambridge, MA, USA). Horseradish peroxidase-conjugated anti-rabbit IgG secondary antibody (Bio-Rad Laboratories, Inc., Hercules, CA, USA) was then added at a dilution of 1:5,000, and proteins were detected by enhanced chemiluminescence using a Western Blotting Luminol reagent (Santa Cruz Biotechnology, Inc., Dallas, TX, USA). The density of the bands was analyzed by Quantity One software version 4.62 (Bio-Rad Laboratories).

Terminal deoxynucleotidyl transferase-mediated dUTP nick end labeling (TUNEL) assay for apoptosis detection. Apoptotic neuronal cells in the hippocampus of the intracerebral hemorrhage animal model were analyzed using TUNEL assay (DeadEnd ${ }^{\mathrm{TM}}$ Colorimetric TUNEL System; Promega Corporation, Madison, WI, USA) according to the manufacturer's protocol. The slides were then analyzed with fluorescence microscopy (Bx51; Olympus Corporation, Tokyo, Japan).

Analysis of cerebral water content $(C W C)$. The CWC was measured on day 10 after treatment with simvastatin following intracerebral hemorrhage as described previously (17). Briefly, the brains of the mice were isolated and divided into the two hemispheres. The hemispheres were weighed using an electronic analytical balance to obtain the wet weights. Subsequently, the brain tissues were dried in an electric oven at $100^{\circ} \mathrm{C}$ for $24 \mathrm{~h}$ to analyze the CWC in the intracerebral hemorrhage mouse model. The $\mathrm{CWC}$ was calculated according to the following formula: CWC $(\%)=($ Wet weight-dry weight $) /$ wet weight $\mathrm{x} 100$.

Quantitative analysis of blood-brain barrier (BBB) permeability. BBB leakage was assessed as previously described with a slight modification (18). Briefly, all experimental mice received $100 \mu \mathrm{l}$ of a 5\% solution of Evans blue (cat. no. E2129; Sigma-Aldrich; Merck KGaA) in simvastatin or saline administered intravenously with the final dose of simvastatin or vehicle at 10 days following intracerebral hemorrhage. At $2 \mathrm{~h}$ following Evans blue injection, cardiac perfusion was performed under deep anesthesia with $200 \mathrm{ml}$ saline to clear the cerebral circulation of the Evans blue. Following sacrifice, the brain was isolated, embedded in paraffin and cut into $20-\mu$ m-thick cryosections. The two hemispheres were homogenized in $750 \mu 1 \mathrm{~N}, \mathrm{~N}$-dimethylformamide (cat. no. D4551; Sigma-Aldrich; Merck KGaA). The samples obtained were incubated in a $50^{\circ} \mathrm{C}$ water bath for $48 \mathrm{~h}$ and subsequently centrifuged at $12,000 \times \mathrm{g}$ for $30 \mathrm{~min}$ at $25^{\circ} \mathrm{C}$. The resultant supernatant was spectrophotometrically quantified for the extravasated Evans blue dye at $620 \mathrm{~nm}$.

$N F-\kappa B$ activity. Neuronal cells in experimental mice were isolated as described above. A total of $1 \times 10^{6}$ cells were cultured in Dulbecco's modified Eagle's medium (Gibco; Thermo Fisher Scientific, Inc.) supplemented with $10 \%$ fetal bovine serum (Hyclone; GE Healthcare Life Sciences, Logan, UT, USA) and incubated at $37^{\circ} \mathrm{C}$ for $12 \mathrm{~h}$. Neuronal cells were transfected with plentivirus (p)-NF- $\kappa$ B-Lucreporter plasmid using the VigoFect Transfection reagent (Vigorous Biotechnology, Beijing, China). A total of $24 \mathrm{~h}$ following transfection, cells were lysed and the $\mathrm{NF}-\kappa \mathrm{B}$ luciferase activity was determined using the Dual-Luciferase Reporter Assay system (Promega Corporation, Madison, WI, USA) according to the manufacturer's protocol. 
A

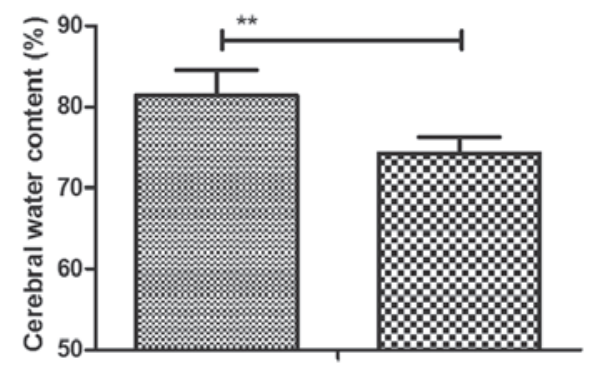

B

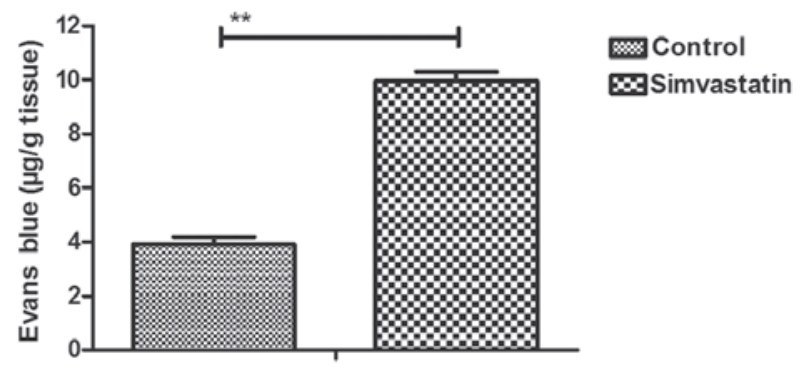

Figure 1. Simvastatin improved the CWC and BBB disruption in intracerebral hemorrhage animals. Simvastatin treatment (A) decreased the CWC and (B) improved the BBB disruption as observed by the increase of the Evans blue content in the intracerebral hemorrhage animals, as compared with the vehicle-treated model mice. ${ }^{* *} \mathrm{P}<0.01$. CWC, cerebral water content; $\mathrm{BBB}$, blood-brain barrier.
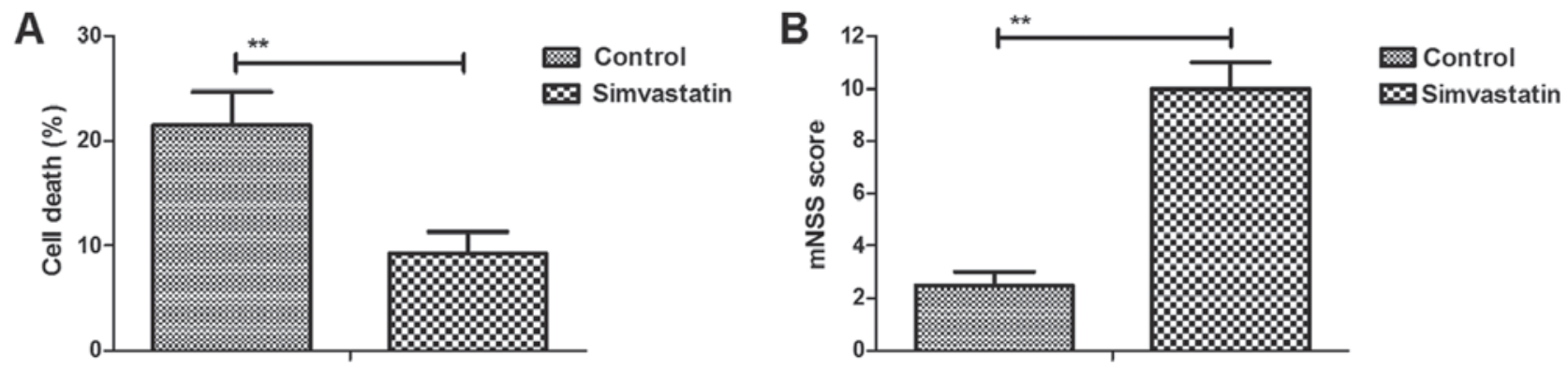

Figure 2. Simvastatin decreased the intracerebral hemorrhage-induced neuronal cell death. (A) Simvastatin treatment significantly reduced the cell death in the injured hemispheres. (B) Simvastatin markedly improved the motor function of the intracerebral hemorrhage animals compared with the vehicle-treated model animals, as observed by the increase in mNSS values. ${ }^{* *} \mathrm{P}<0.01$. mNSS, modified neurological severity score.

$N F-\kappa B$ overexpression. Neuronal cells $\left(1 \times 10^{5}\right)$ were cultured in 6 -well culture plates until $85 \%$ confluence, when the media was removed and the plates were washed three times with PBS. Neuronal cells were transfected with $\mathrm{pNF}-\kappa \mathrm{B}$ using Lipofectamine ${ }^{\circledR} 2000$ (Sigma-Aldrich; Merck KGaA) according to manufacturer's protocol. Cells with NF- $\kappa \mathrm{B}$ overexpression were treated with simvastatin $(200 \mu \mathrm{M})$ for further analysis.

Behavioral assessments. Animal behavioral assessment was performed on postoperative day 10 . The assessment parameters, including the left limb movement and coordination of movement, were evaluated using the modified neurological severity score (mNSS) as previously described (19). Each animal in the present study was analyzed using mNSS scores. The total possible score was 15 , mNSS scores of $\geq 10$ indicated normal behavior, scores $\geq 5$ indicated behavioral difficulties and $\mathrm{mNSS}$ scores $<5$ indicated dysfunctional behavior.

Statistical analysis. Data are presented as the mean \pm standard deviation of triplicate experiments. All data were analyzed by SPSS version 13.0 software (SPSS, Inc., Chicago, IL, USA). Comparisons between the groups were assessed by Student's t-test or one-way analysis of variance. A P-value of $<0.05$ was considered to indicate a statistically significant result.

\section{Results}

Simvastatin improves the $C W C$ and $B B B$ disruption in intracerebral hemorrhage animals. An intracerebral hemorrhage mouse model was used to analyze the efficacy of simvastatin treatment. It was observed that simvastatin treatment significantly decreased the CWC in intracerebral hemorrhage animals compared with the vehicle-treated model group (Fig. 1A). The results also revealed that simvastatin treatment improved BBB disruption in the intracerebral hemorrhage animals compared with the vehicle-treated model group, as observed by the marked increase in the Evans blue content of brain tissues (Fig. 1B). These results suggested that simvastatin treatment significantly improved the $\mathrm{CWC}$ and $\mathrm{BBB}$ disruption in intracerebral hemorrhage animals.

Simvastatin decreases intracerebral hemorrhage-induced neuronal cell death. The efficacy of simvastatin on neuronal cell death was investigated in intracerebral hemorrhage mice. As shown in Fig. 2A, simvastatin treatment significantly reduced cell death in the injured hemispheres. Furthermore, it was demonstrated that simvastatin markedly improved the motor function in intracerebral hemorrhage animals compared with the vehicle-treated model group, as observed by the marked increase in mNSS values (Fig. 2B). These results suggested that simvastatin decreased the intracerebral hemorrhage-induced neuronal cell death.

Simvastatin decreases the apoptosis of neurons in intracerebral hemorrhage animals. The anti-apoptotic effects of simvastatin were analyzed in intracerebral hemorrhage animals, and it was observed that simvastatin treatment significantly decreased the apoptosis of neuronal cells in 
A
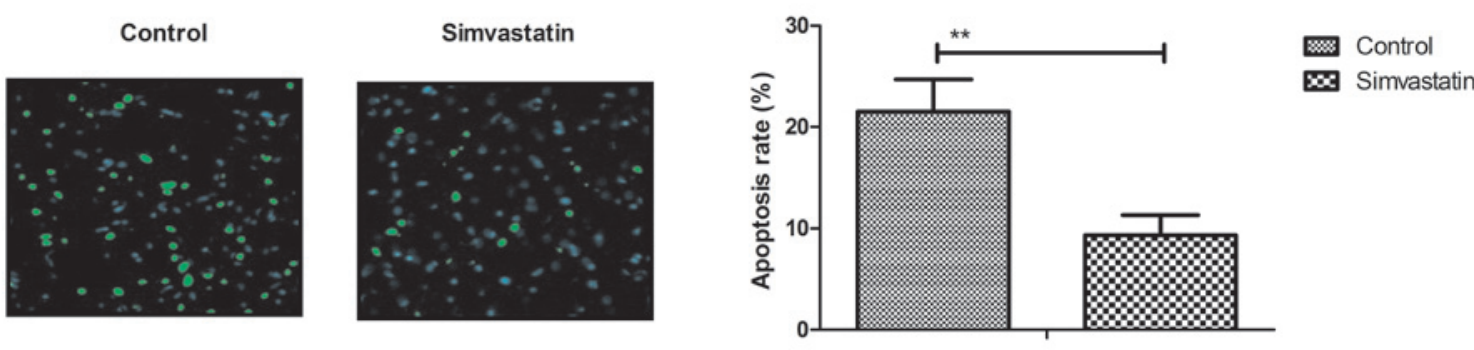

B
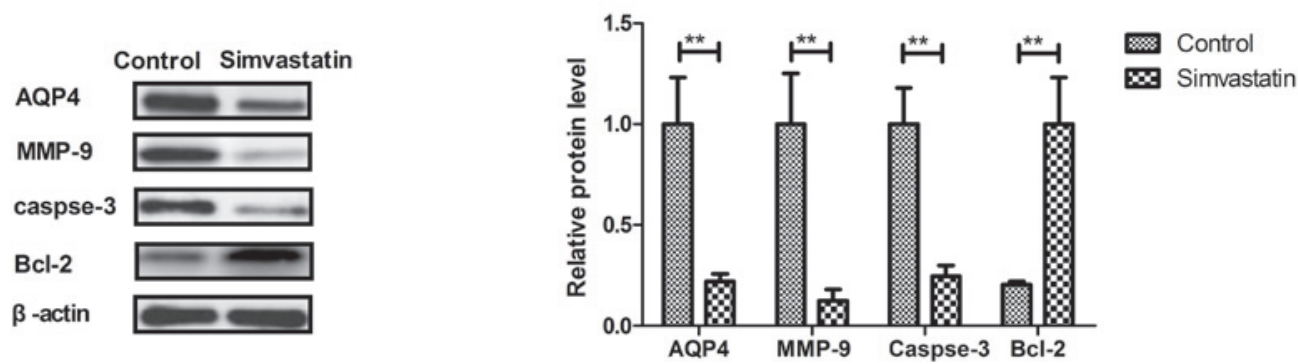

Figure 3. Simvastatin decreased the apoptosis of neurons in the intracerebral hemorrhage animals. (A) A TUNEL assay and the quantified apoptotic rate indicated a reduced rate of apoptosis in simvastatin-treated animals. (B) Western blot analysis of the expression levels of AQP4, MMP-9, caspase-3 and Bcl-2 in neuronal cells in experimental mice. Simvastatin treatment significantly downregulated the expression levels of AQP4, MMP-9 and caspase-3 Whereas it significantly upregulated the protein expression of Bcl-2. ${ }^{* *} \mathrm{P}<0.01$. AQP4, aquaporin-4; MMP-9, matrix metallopeptidase 9; Bcl-2, B-cell lymphoma-2.

A
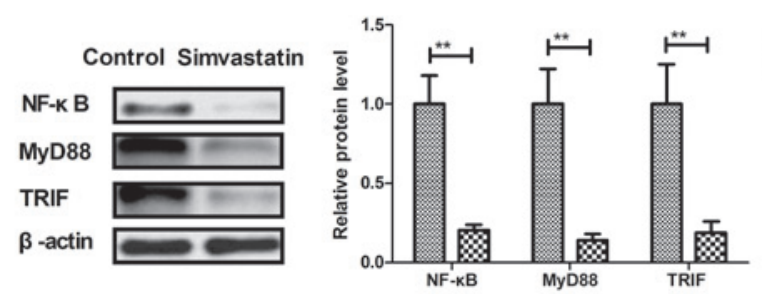

C
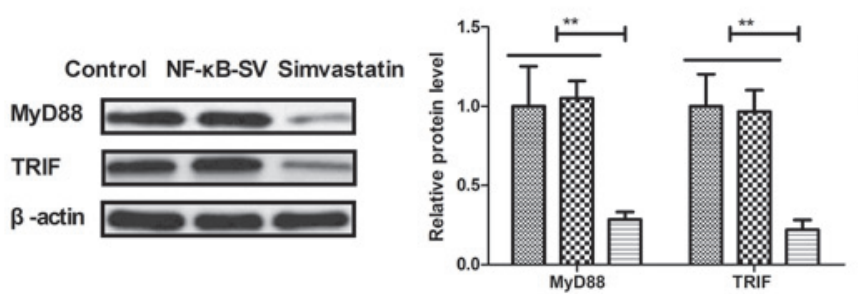

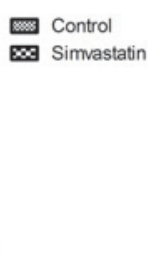

B

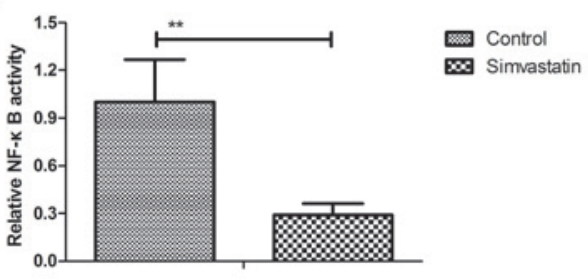

D

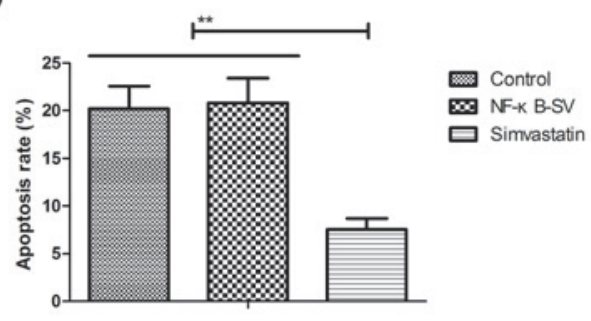

Figure 4. Simvastatin reduced cellular apoptosis by suppressing the NF-kB-mediated MyD88/TRIF signaling pathway. (A) Western blot analysis demonstrated that simvastatin treatment downregulated the NF- $\mathrm{kB}, \mathrm{MyD} 88$ and TRIF expression levels in neuronal cells in the experimental mice. (B) NF- $\mathrm{kB}$ activity was decreased by simvastatin in neuronal cells in experimental mice. (C) Western blot analysis revealed that NF- $\mathrm{kB}$ overexpression abolished the simvas-

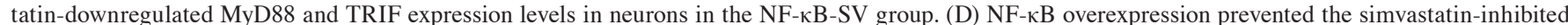

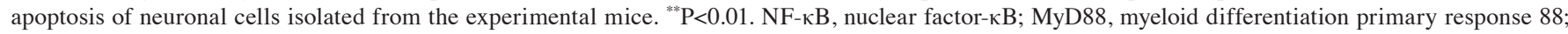
TRIF, TIR domain-containing adaptor protein inducing interferon- $\beta$. NF- $\kappa B S V, N F-\kappa B$ overexpression + simvastatin.

intracerebral hemorrhage animals (Fig. 3A). In addition, the expression levels of AQP4, MMP-9 and caspase-3 were downregulated and Bcl-2 were all upregulated by simvastatin treatment compared with the levels in the vehicle-treated model group (Fig. 3B). These results suggest that simvastatin treatment can decrease the apoptosis of neurons in animals suffering intracerebral hemorrhage.
Simvastatin reduced cellular apoptosis by suppressing $N F$ - $\kappa B$-mediated MyD88/TRIF. The potential mechanism underlying the effect of simvastatin in neurons in the intracerebral hemorrhage animals was also examined in the present study. Mechanism analysis indicated that simvastatin treatment significantly downregulated the NF- $\mathrm{BB}, \mathrm{MyD} 88$ and TRIF expression levels in the neuronal cells in experimental 
mice as compared with the vehicle-treated model mice (Fig. 4A). Furthermore, the NF- $\kappa$ B activity was also decreased by simvastatin in the neuronal cells of the experimental mice (Fig. 4B).

The results also demonstrated that NF- $\kappa \mathrm{B}$ overexpression abolished simvastatin-downregulated MyD88 and TRIF expression in the neurons of the NF- $\mathrm{NB}+$ simvastatin group (Fig. 4C). Simvastatin-inhibited apoptosis was also prevented by the $\mathrm{NF}-\kappa \mathrm{B}$ overexpression in neuronal cells (Fig. 4D). These results suggested that simvastatin treatment reduced cellular apoptosis by suppressing the NF- $\kappa \mathrm{B}$-mediated MyD88/TRIF signaling pathway in the neurons of the intracerebral hemorrhage animals.

\section{Discussion}

Intracerebral hemorrhage is frequently induced by exertion and emotion, and the majority of patients demonstrate sudden onset during activity. Previous evidence revealed that simvastatin exhibits beneficial effects for patients with intracerebral hemorrhage (20). In addition, simvastatin has been demonstrated to attenuate cerebral vasospasm and improve the treatment outcomes by upregulation of various signaling pathways in a rat model of subarachnoid hemorrhage (21). In the present study, the potential mechanism mediated by simvastatin was analyzed in a CD-1 mouse model of intracerebral hemorrhage. It was demonstrated that simvastatin treatment significantly improved the CWC and BBB disruption in this mouse model. The findings also suggested that intracerebral hemorrhage-induced neuronal cell death was decreased by the simvastatin treatment. Notably, simvastatin treatment significantly reduced the Evans blue leakage into the injured hemispheres and improved the motor function. Mechanism analysis indicated that simvastatin treatment was able to reduce cellular apoptosis by suppressing the NF- $\kappa \mathrm{B}$-mediated MyD88/TRIF signaling pathway subsequent to intracerebral hemorrhage in mice.

Subarachnoid hemorrhage is one of the most severe cerebral hemorrhage types and usually leads to mortality due to bleeding into the subarachnoid space $(22,23)$. However, simvastatin treatment was observed to attenuate the cerebral vasospasm following subarachnoid hemorrhage in rats via increased phosphorylation of protein kinase B and endothelial nitric oxide synthase (24). Lin et al (25) have also demonstrated the therapeutic effects of simvastatin on delayed cerebral vasospasm following subarachnoid hemorrhage in rabbits. In the present study, simvastatin treatment markedly improved CWC and BBB disruption, which further improved the motor function in intracerebral hemorrhage animals.

The preventive and therapeutic effects of simvastatin on secondary inflammatory damage have previously been investigated in rats with cerebral hemorrhage (26). Another study observed that NF- $\kappa \mathrm{B}$ activation was closely associated with cell death and served an important function in secondary brain damage following intracerebral hemorrhage in patients (27). Furthermore, Shen et al (28) indicated that $\mathrm{NF}-\kappa \mathrm{B}$ regulates the intracerebral hemorrhage-induced neuronal damage via apoptosis. A further study also indicated that simvastatin reduces the $\mathrm{NF}-\kappa \mathrm{B}$ activity in peripheral mononuclear and in plaque cells of rabbit atheroma (29). In the current investigation, it was demonstrated that simvastatin treatment downregulated the $\mathrm{NF}-\kappa \mathrm{B}$ expression, and upregulated the MyD88 and TRIF expression levels in neuronal cells in the experimental mice. In addition, $\mathrm{NF}-\kappa \mathrm{B}$ overexpression abolished the simvastatin-induced downregulation of MyD88 and TRIF levels in neurons.

In conclusion, the present study reported the therapeutic effects of simvastatin on experimental mice with intracerebral hemorrhage. The anti-apoptotic efficacy of simvastatin on neurons in the progression of intracerebral hemorrhage was also discussed. The study findings indicated that simvastatin treatment attenuated brain edema and reduced cellular apoptosis by suppressing the NF- $\kappa \mathrm{B}-$ mediated MyD88/TRIF signaling pathway following intracerebral hemorrhage in mice. These data suggest that simvastatin is an efficient drug for intracerebral hemorrhage therapy and identified that $\mathrm{NF}-\kappa \mathrm{B}$ may be a potential target for the treatment of intracerebral hemorrhage.

\section{References}

1. Sussman ES and Connolly ES Jr: Hemorrhagic transformation: A review of the rate of hemorrhage in the major clinical trials of acute ischemic stroke. Front Neurol 4: 69, 2013.

2. Ma B and Zhang J: Nimodipine treatment to assess a modified mouse model of intracerebral hemorrhage. Brain Res 1078: 182-188, 2006.

3. Manaenko A, Fathali N, Chen H, Suzuki H, Williams S, Zhang JH and Tang J: Heat shock protein 70 upregulation by geldanamycin reduces brain injury in a mouse model of intracerebral hemorrhage. Neurochem Int 57: 844-850, 2010.

4. Lee HJ, Kim KS, Kim EJ, Choi HB, Lee KH, Park IH, Ko Y, Jeong SW and Kim SU: Brain transplantation of immortalized human neural stem cells promotes functional recovery in mouse intracerebral hemorrhage stroke model. Stem Cells 25: 1204-1212, 2007

5. Ganesh Kumar N, Zuckerman SL, Khan IS, Dewan MC, Morone PJ and Mocco J: Treatment of intracerebral hemorrhage: A selective review and future directions. J Neurosurg Sci 61: 523-535, 2017.

6. Lee WJ, Yeon JY, Jo KI, Kim JS and Hong SC: Reversible cerebral vasoconstriction syndrome and posterior reversible encephalopathy syndrome presenting with deep intracerebral hemorrhage in young women. J Cerebrovasc Endovasc Neurosurg 17: 239-245, 2015 .

7. Kanamaru K, Suzuki H and Taki W: Cerebral infarction after aneurysmal subarachnoid hemorrhage. Acta Neurochir Suppl 121: 167-172, 2016.

8. Lu L, Barfejani AH, Qin T, Dong Q, Ayata C and Waeber C: Fingolimod exerts neuroprotective effects in a mouse model of intracerebral hemorrhage. Brain Res 1555: 89-96, 2014.

9. Ramadan WH and Kabbara WK: Sitagliptin/simvastatin: A first combination tablet to treat type 2 diabetes and hypercholesterolemia-a review of its characteristics. Vasc Health Risk Manag 11: $125-132,2015$

10. Taveira-DaSilva AM, Jones AM, Julien-Williams PA, Stylianou M and Moss J: Retrospective review of combined sirolimus and simvastatin therapy in lymphangioleiomyomatosis. Chest 147: 180-187, 2015.

11. Yasuda $H$, Yuen PS, Hu X, Zhou $H$ and Star RA: Simvastatin improves sepsis-induced mortality and acute kidney injury via renal vascular effects. Kidney Int 69: 1535-1542, 2006.

12. Karki K, Knight RA, Han Y, Yang D, Zhang J, Ledbetter KA, Chopp M and Seyfried DM: Simvastatin and atorvastatin improve neurological outcome after experimental intracerebral hemorrhage. Stroke 40: 3384-3389, 2009.

13. Lapchak PA and Han MK: The 3-hydroxy-3-methylglutaryl coenzyme A reductase inhibitor simvastatin reduces thrombolytic-induced intracerebral hemorrhage in embolized rabbits. Brain Res 1303: 144-150, 2009.

14. Yang D, Knight RA, Han Y, Karki K, Zhang J, Ding C, Chopp M and Seyfried DM: Vascular recovery promoted by atorvastatin and simvastatin after experimental intracerebral hemorrhage: Magnetic resonance imaging and histological study. J Neurosurg 114: 1135-1142, 2011. 
15. Shimamura N, Kakuta K, Wang L, Naraoka M, Uchida H, Wakao S, Dezawa M and Ohkuma H: Neuro-regeneration therapy using human Muse cells is highly effective in a mouse intracerebral hemorrhage model. Exp Brain Res 235: 565-572, 2017.

16. Park JS, Kim S, Han DK, Lee JY and Ghil SH: Isolation of neural precursor cells from skeletal muscle tissues and their differentiation into neuron-like cells. Exp Mol Med 39: 483-490, 2007.

17. Hijioka M, Matsushita H, Hisatsune A, Isohama $\mathrm{Y}$ and Katsuki H: Therapeutic effect of nicotine in a mouse model of intracerebral hemorrhage. J Pharmacol Exp Ther 338: 741-749, 2011.

18. Tong LS, Shao AW, Ou YB, Guo ZN, Manaenko A, Dixon BJ, Tang J, Lou M and Zhang JH: Recombinant Gas6 augments Axl and facilitates immune restoration in an intracerebral hemorrhage mouse model. J Cereb Blood Flow Metab 37: 1971-1981, 2017.

19. Hsieh TH, Kang JW, Lai JH, Huang YZ, Rotenberg A, Chen KY, Wang JY, Chan SY, Chen SC, Chiang YH and Peng CW: Relationship of mechanical impact magnitude to neurologic dysfunction severity in a rat traumatic brain injury model. PLoS One 12: e0178186, 2017.

20. McGirt MJ, Pradilla G, Legnani FG, Thai QA, Recinos PF Tamargo RJ and Clatterbuck RE: Systemic administration of simvastatin after the onset of experimental subarachnoid hemorrhage attenuates cerebral vasospasm. Neurosurgery 58: 945-951, 2006.

21. Sugawara T, Jadhav V, Ayer R and Zhang J: Simvastatin attenuates cerebral vasospasm and improves outcomes by upregulation of PI3K/Akt pathway in a rat model of subarachnoid hemorrhage. Acta Neurochir Suppl 102: 391-394, 2008.
22. Brown RJ, Epling BP, Staff I, Fortunato G, Grady JJ and McCullough LD: Polyuria and cerebral vasospasm after aneurysmal subarachnoid hemorrhage. BMC Neurol 15: 201, 2015.

23. Jenson AV, Rodriguez GJ, Alvarado LA, Cruz-Flores S and Maud A: Higher rate of intracerebral hemorrhage in hispanic patients with cerebral cavernous malformation. J Vasc Interv Neurol 8: 1-4, 2015.

24. Sugawara T, Ayer R, Jadhav V, Chen W, Tsubokawa T and Zhang JH: Simvastatin attenuation of cerebral vasospasm after subarachnoid hemorrhage in rats via increased phosphorylation of Akt and endothelial nitric oxide synthase. J Neurosci Res 86: 3635-3643, 2008.

25. Lin C, Zhao Y, Wan G, Zhu A and Wang H: Effects of simvastatin and taurine on delayed cerebral vasospasm following subarachnoid hemorrhage in rabbits. Exp Ther Med 11: 1355-1360, 2016.

26. Zhou HX, Gao LH, Meng LL, Zhang YX, Wei ZF and Si DW: Preventive and therapeutic effect of simvastatin on secondary inflammatory damage of rats with cerebral hemorrhage. Asian Pac J Trop Med 10: 152-156, 2017.

27. Zhang Z, Liu Y, Huang Q, Su Y, Zhang Y, Wang G and Li F. NF-kB activation and cell death after intracerebral hemorrhage in patients. Neurol Sci 35: 1097-1102, 2014.

28. Shen X, Ma L, Dong W, Wu Q, Gao Y, Luo C, Zhang M, Chen X and Tao L: Autophagy regulates intracerebral hemorrhage induced neural damage via apoptosis and NF-kB pathway. Neurochem Int 96: 100-112, 2016.

29. Mikael LG and Rozen R: Homocysteine modulates the effect of simvastatin on expression of ApoA-I and NF-kappaB/iNOS. Cardiovasc Res 80: 151-158, 2008. 